\title{
Relationships Between Conductor Damage, Quenching and Electromechanical Behavior in YBCO Coated Conductors
}

\author{
A. L. Mbaruku, U. P. Trociewitz, X. Wang, Student Member, IEEE, and J. Schwartz, Fellow, IEEE
}

\begin{abstract}
The implementation of emerging superconducting materials into magnet systems with long service lifetimes requires a thorough understanding of their engineering properties, including their quench and electromechanical behaviors. Furthermore, it is essential to understand the role of defects in the conductor, whether they be pre-existing defects from the conductor manufacturing process that locally reduce $J_{c}$, or local defects that result from a non-destructive quench (i.e., a quench that may reduce $J_{c}$ locally but does not significantly affect the end-to-end behavior). This paper reports results on both of these types of defects and the interplay between quenching and electromechanical behavior. Quench studies investigate the initiation and propagation of quenches in coated conductors. Disturbances in homogeneous conductors are initiated by a pulsed heater attached to the conductor. Disturbances in locally damaged conductors are initiated by increasing the transport current above the $I_{c}$ at the local defect but below the end-to-end $I_{c}$. Samples are quenched to determine the minimum quench energy and the quench propagation velocity. Homogeneous samples are also quenched to the point of initiating local damage, thereby identifying the maximum allowable hot-spot temperature or hot-spot temperature gradient. Samples used in quench studies are subsequently used in $I_{c}$-strain measurements to determine how quenching affects subsequent performance. Samples that exhibit reduced $I_{c}$ from quenching, and samples from regions adjacent to such damaged samples, are studied. It is found that quenching can reduce the electromechanical performance of conductors that do not initially show a reduction in their electrical performance.
\end{abstract}

Index Terms-Coated conductor, electromechanical behavior, quench damage effects.

\section{INTRODUCTION}

$\mathbf{Q}$ UENCHING of superconducting magnets is not desired in any operation. While high-temperature superconductor based magnets may have higher stability margin, the slow quench propagation velocity, on the order of $\mathrm{mm} / \mathrm{s}$, may

Manuscript received August 29, 2006. This work was supported in part by the Air Force Office of Scientific Research, the National High Magnetic Field Laboratory (NHMFL), and the Center for Advanced Power Systems (CAPS).

A. L. Mbaruku and U. P. Trociewitz are with the National High Magnetic Field Laboratory, Tallahassee, FL 32310 USA (e-mail: mbaruku@ magnet.fsu. edu; trociew@magnet.fsu.edu).

X. Wang is with the FAMU-FSU College of Engineering, Tallahassee, FL 32310 USA, and the Center for Advanced Power Systems, Tallahassee, FL 32310 USA (e-mail: xiarong@ @ail.caps.fsu.edu).

J. Schwartz is with the National High Magnetic Field Laboratory, Tallahassee, FL 32310 USA. He is also with the FAMU-FSU College of Engineering, Tallahassee, FL 32310 USA (e-mail: schwartz@magnet.fsu.edu).

Color versions of one or more of the figures in this paper are available online at http://ieeexplore.ieee.org.

Digital Object Identifier 10.1109/TASC.2007.898874

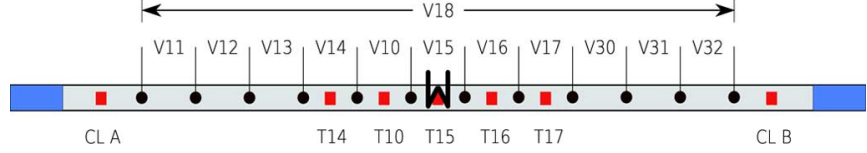

Fig. 1. Schematic of voltage taps, thermocouples and the NiCr heater attached to the sample during quench experiments. The solid squares are thermocouples and the solid rectangles at each end are current leads.

make them vulnerable to excessive heating and damage if subjected to a quench [1]. If a magnet is effectively protected, then quenching does not damage the superconductor. Previous studies have shown, however, that YBCO is susceptible to damage in the event of a magnet quench. Degradation of $I_{c}$ was observed when YBCO coated conductors were quenched in liquid nitrogen with over-current pulses [2], [3]; clearly the quench protection parameters must be understood. Furthermore, because the failure of high temperature superconductors is fundamentally different than metallic low temperature superconductors, it is possible that a quench can appear to not cause any damage (i.e., not reduce $I_{c}$ ), but in fact alter the electro-mechanical properties of the conductor. This work reports results on the effects of quenching on electromechanical behavior for YBCO coated conductors.

\section{EXPERIMENTS}

\section{A. Samples}

All samples for this study were derived from the same batch of tape manufactured and supplied by IGC Superpower Inc. The superconducting YBCO layer was coated on IBAD-YSZ and on $100 \mu \mathrm{m}$ thick Hastelloy C-276 substrate. The $1.2 \mathrm{~cm}$ wide tape was slit into $4 \mathrm{~mm}$ wide tapes and electroplated with $36 \mu \mathrm{m}$ thick Cu stabilizer. The tape has a $3 \mu \mathrm{m}$ thick Ag layer, a $1.4 \mu \mathrm{m}$ thick YBCO layer and $1 \mu \mathrm{m}$ thick buffer layer.

\section{B. Partial Quench Damage Induction}

Controlled partial quench-damage causes $I_{c}$ degradation in some sections of $180 \mathrm{~mm}$ long samples. Voltage taps and thermocouples monitor the voltage and temperature versus time during the experiment. Fig. 1 shows a schematic diagram of the wiring on the sample. Each voltage section is $10 \mathrm{~mm}$ long and type $\mathrm{E}$ thermocouples are attached in the middle of each voltage section. A NiCr wire heater is attached to the YBCO facing side using Stycast 2850FT. Samples are cryocooled to $37 \mathrm{~K}$; after cooling the pressure within the cryostat is $10^{-6}$ mbar. More experimental details are found in [4], [5]. 
The voltage across each section is recorded while ramping the transport current using a fixed ramp rate controlled by a home-made ramp generator and a HP 6881A power supply. $I_{c}$ is determined by a $1 \mu \mathrm{V} / \mathrm{cm}$ electric field criterion and the V-I curve is fit by an exponential function to determine the n-value. More details on $I_{c}$ measurements is found in [6].

Prior to any other experiments, the $I_{c}$ versus location is measured. A steady-state transport current of $160 \mathrm{~A}$, corresponding to $70 \%$ of the end-to-end $I_{c}$, is established in the conductor. A quench is then induced by pulsing the heater, locally raising the sample temperature. This process is repeated with the heat pulse duration varied until the local voltage versus time in some section of the sample does not recover, indicating a quench. The transport current is then stopped and the sample recooled to the original temperature. The $I_{c}$ distribution is then remeasured to determine if the sample is damaged. If the sample is damaged, the damaged sections are identified and separated from the undamaged sections.

\section{Stress-Strain, Strain- $I_{c}$ and Fatigue- $I_{c}$ Tests}

Stress-strain, $I_{C}$-strain and $I_{c}$-fatigue tests are performed using a low temperature electromechanical testing (LTET) device developed at the NHMFL and described in detail in [7], [8]. The tests are conducted at $77 \mathrm{~K}$ on partially quench damaged and non-damaged samples. A $55 \mathrm{~mm}$ long sample is mounted on the LTET while ensuring that there is sufficient slack to avoid tensioning the sample during cooling down.

For $I_{c}$-strain measurements, $I_{c}$ is first measured in the as-cooled condition and at the onset of load. The strain is then increased in $\sim 0.015 \%$ steps and $I_{C}$ remeasured after each step. The experiment is continued until $I_{c}<7 \mathrm{~A}$.

For $I_{c}$-fatigue tests, the samples are subjected to strain controlled fatigue with a maximum strain of $0.35 \%$ and a strain ratio of 0.5 with a $0.4 \mathrm{~Hz}$ loading frequency. $I_{c}$ is measured after 1 , 10,100 and 1000 cycles, and subsequently in 6000 cycle intervals. The experiment is continued until the number of cycles reached $2 \times 10^{5}$. Samples that have not failed after $2 \times 10^{5} \mathrm{cy}-$ cles are subjected to $I_{C}$-strain tests.

\section{Microstructural Observations}

Sample microstructures are studied using an environmental scanning electron microscope (ESEM). To observe the YBCO layer, it is necessary to first remove the $\mathrm{Cu}$ and $\mathrm{Ag}$ layers. The copper layer is etched using diluted nitric acid $\left(30 \%\right.$ vol. $\mathrm{HNO}_{3}$ : $70 \%$ vol. $\mathrm{H}_{2} \mathrm{O}$ ). The samples are then rinsed in water and the $\mathrm{Ag}$ etched using a solution of $25 \%$ vol. $\mathrm{H}_{2} \mathrm{O}_{2}: 25 \%$ vol. $\mathrm{NH}_{4} \mathrm{OH}$ : $50 \%$ vol. $\mathrm{H}_{2} \mathrm{O}$ that is freshly made for each sample. Precaution was taken to ensure that the etching process only occurs in the desired area and always from the top surface of the sample and not from the edges. This was done by applying a thin layer of nail varnish on all edges and surfaces where etching is to be prevented.

\section{RESULTS}

The quench behavior of the sample was extensively tested before inducing damage. Thus, the sample experienced 164 heater pulses of varying duration to determine the minimum quench energy and normal zone propagation velocity at $40 \mathrm{~K}, 50 \mathrm{~K}$, $60 \mathrm{~K}, 70 \mathrm{~K}$ and $77 \mathrm{~K}$. During these experiments, the sample temperature is limited by terminating the experiments shortly

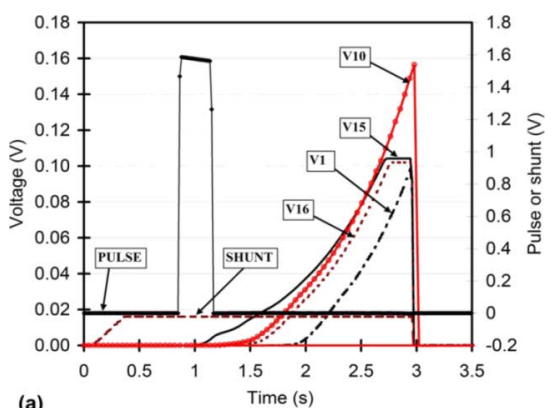

(a)

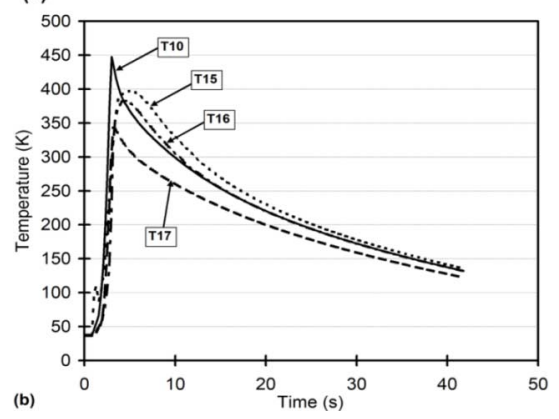

Fig. 2. (a) Voltage and (b) temperature versus time during the quench experiment resulting in partial damage to the conductor.

after determining if the sample had quenched. Of the 164 heat pulses (quench attempts), the sample quenched 77 times and recovered 87 times. The sample frequently reached a temperature of $150 \mathrm{~K}$ and experienced a peak temperature of $250 \mathrm{~K}$ during one quench; no measurable change in the $I_{c}$ profile resulted, indicating that YBCO conductors can be protected such that quenching does not always cause damage.

To simulate an unprotected quench, a severe quench is initiated by a heat pulse and the transport current is maintained for about $2 \mathrm{~s}$ so that the highest temperature on the tape rises to about $450 \mathrm{~K}$, as shown in Fig. 2. Fig. 3 shows the $I_{c}$ profile measured before and after the severe quench. The event caused a $\sim 50 \%$ reduction in $I_{c}$ in section V10 which corresponds to the highest temperature in the tape. This is also the section the lowest initial $I_{c}$. Section V15, where the heater was located, experienced a peak temperature of $\sim 400 \mathrm{~K}$ and its $I_{c}$ decreased by $33 \%$ to $160 \mathrm{~A}$. Sections away from the hot spot have less or no obvious $I_{c}$ degradation. While these sections remain intact, the local degradation at sections V10 and V15 cause the tape end-to-end $I_{c}$ (V18) to be reduced by $\sim 41 \%$ to $130 \mathrm{~A}$.

Fig. 4 shows stress-strain results for the non-damaged and partially quench-damaged samples. Initially, the samples behave similarly, but as the stress increases above $\sim 250 \mathrm{MPa}$, the properties diverge. The Young's moduli, which are obtained from the initial slopes of the stress-strain curves, are $150 \mathrm{GPa}$ for both samples. The $0.2 \%$ offset yield stresses are $535 \mathrm{MPa}$ and $620 \mathrm{MPa}$ for the partially quench-damaged and non-damaged samples respectively.

$I_{c}$-strain results are shown in Figs. 5-7. Fig. 5 shows that the effect of the partial quench damage on $I_{c}$ is clearly visible. At zero strain, the $I_{c}$ values for the partially quench-damaged and non-damaged samples are 14.73 A and 40.73 A respectively, and the strain at which further $I_{c}$ degradation occurs is $\sim 0.4 \%$.

Fig. 6 shows the $I_{c}$-strain results with $I_{c}$ s normalized to their respective zero strain values $\left(I_{c 0}\right)$. Superconductor failure under applied strain is generally defined as $I_{c}<95 \% I_{c 0}$, shown in 


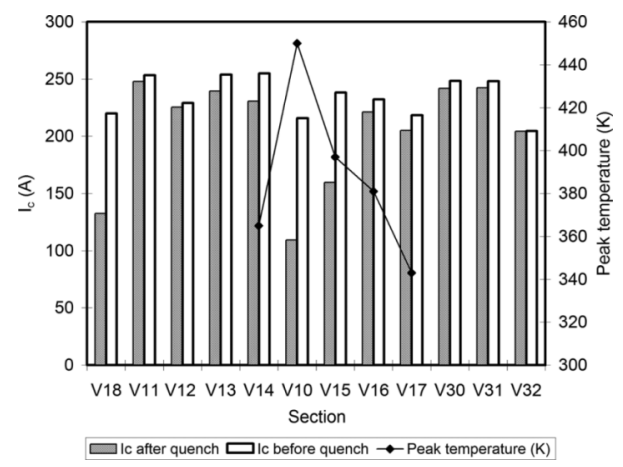

Fig. 3. $I_{c}$ versus location before (open bars) and after (solid bars) the damageinducing quench. The diamonds plot the peak temperature on the tape surface as measured by the thermocouples [the peaks in Fig. 2(b) above].

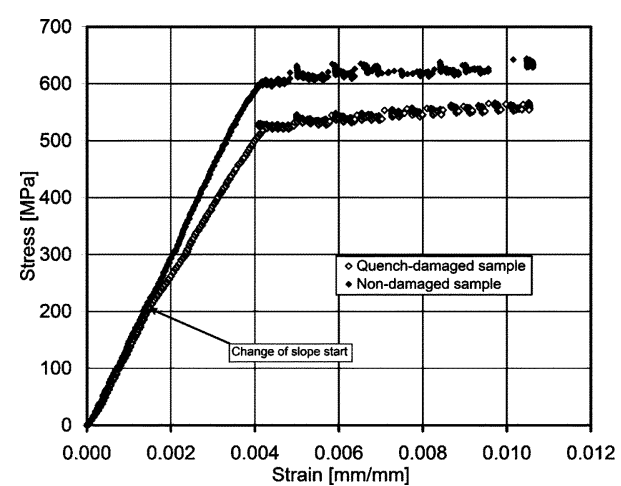

Fig. 4. Stress-strain curves non-damaged and partially quench damaged samples. Young's moduli for both samples are $\sim 150 \mathrm{GPa}$. Quench damage affected the yield stress: $620 \mathrm{MPa}$ for non-damaged and $535 \mathrm{MPa}$ for quench damaged sample.

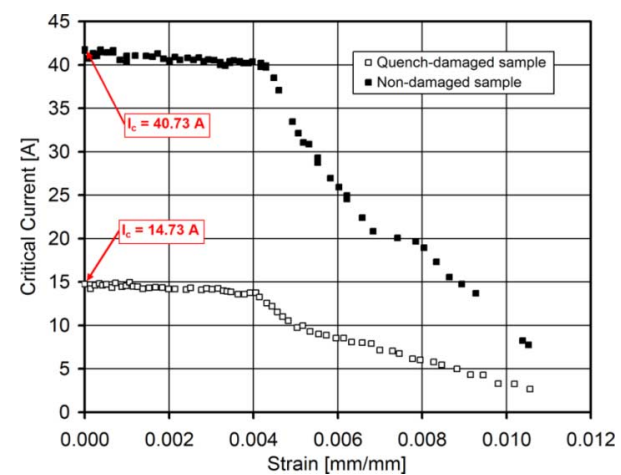

Fig. 5. $I_{c}$ versus strain for non-damaged and partially quench damaged samples. At zero strain, $I_{c}$ is $40.73 \mathrm{~A}$ for the non-damaged sample and $14.73 \mathrm{~A}$ for the partially damaged sample.

the figure by the arrows. Fig. 7 shows the $n$-value versus strain during $I_{c}$-strain testing. The initial $n$-values are 14.7 and 27.8 for the partially quench damaged and non-damaged samples, respectively. In both cases, the $n$-value systematically decreases for strain above 0.004 , with a more rapid decrease occurring in the non-damaged sample.

The results for $I_{c}$-fatigue are shown in Figs. 8 and 9. Fig. 8 shows the normalized $I_{c}$ versus number of cycles $(\mathrm{N})$. The critical currents before fatigue testing were $21.48 \mathrm{~A}$ and $34.89 \mathrm{~A}$ for the damaged and non-damaged samples, respectively. Fig. 9 shows $n$-value versus $\mathrm{N}$. The initial $n$-values are 12.0 and 22.3

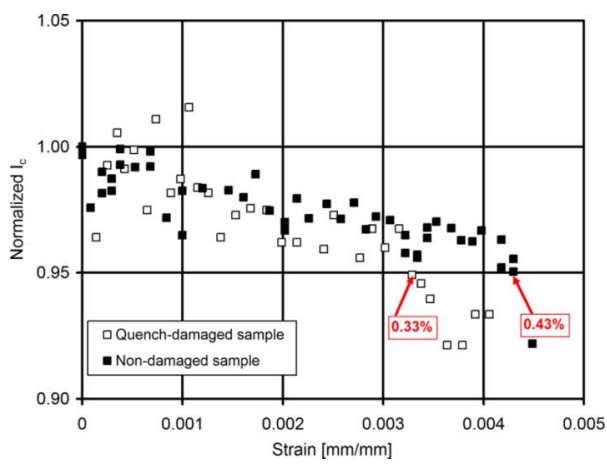

Fig. 6. Normalized $I_{c}$ versus strain for non-damaged and partially quench damaged samples. The arrows indicate the strain at which $I_{c}<95 \% I_{c 0}$.

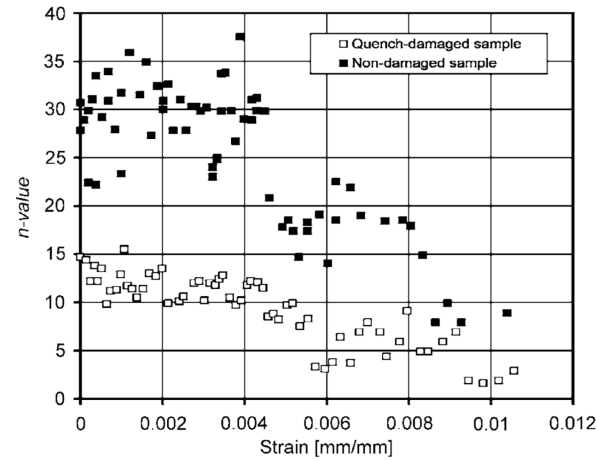

Fig. 7. n-value versus strain for non-damaged and partially quench damaged samples.

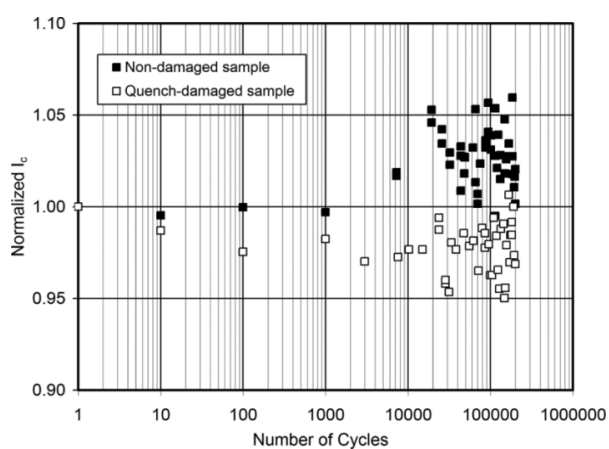

Fig. 8. Normalized $I_{c}$ versus number of cycles for non-damaged and partially quench damaged samples subjected to $2 \times 10^{5}$ fatigue cycles. Fatigue testing was performed at $77 \mathrm{~K}$ with a strain ratio of 0.5 and a maximum strain of $0.35 \%$. Initial $I_{c} \mathrm{~s}$ were $34.89 \mathrm{~A}$ and $21.48 \mathrm{~A}$ for the non-damaged and damaged samples respectively.

for the damaged and non-damaged samples respectively. Fig. 9 also shows that the $n$-values decrease after $\sim 10^{5}$ cycles for both samples. Interestingly, $I_{c}$ and $n$-value for the non-damaged sample show an increase after about $2 \times 10^{4}$ cycles; in the case of $I_{c}$ the increase is only about $5 \%$, which is within experimental error. For $n$-value, however, the increase is as much as $15 \%$.

Fig. 10 shows $I_{c} / I_{c 0}$ versus strain for samples that were previously subjected to $2 \times 10^{5}$ fatigue cycles. The arrows point to the locations where $I_{c}$ drops below $95 \% I_{c 0}$. At zero strain, the $I_{c} \mathrm{~S}$ are $21.43 \mathrm{~A}$ and $34.22 \mathrm{~A}$ for partially quench damaged and non-damaged samples, respectively. Fig. 11 shows the corresponding $n$-value versus strain. The $n$-values at zero strain are 


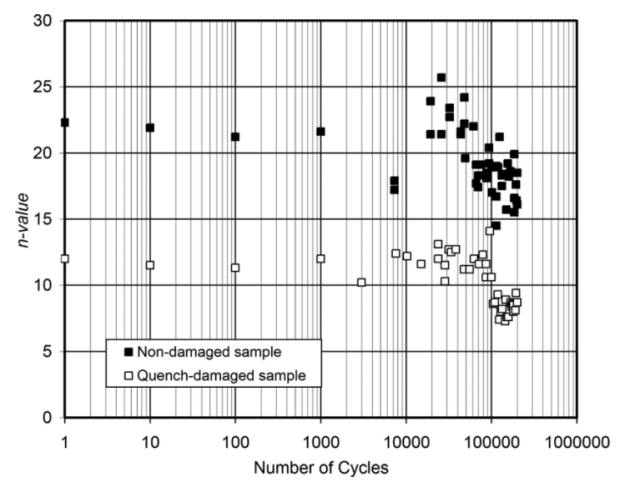

Fig. 9. $n$-value versus number of cycles during $I_{c}$-fatigue testing for quench damaged and non-damaged samples.

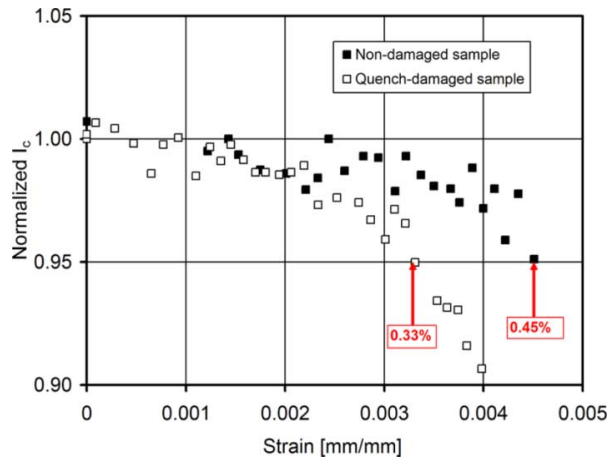

Fig. 10. Normalized $I_{c}$ versus strain for samples previously subjected to $2 \times$ $10^{5}$ fatigue cycles. The initial $I_{c}$ are $21.43 \mathrm{~A}$ and $34.22 \mathrm{~A}$ for the damaged and non-damaged samples respectively. The arrows point to where $I_{c}$ drops below $95 \% I_{c 0}$.

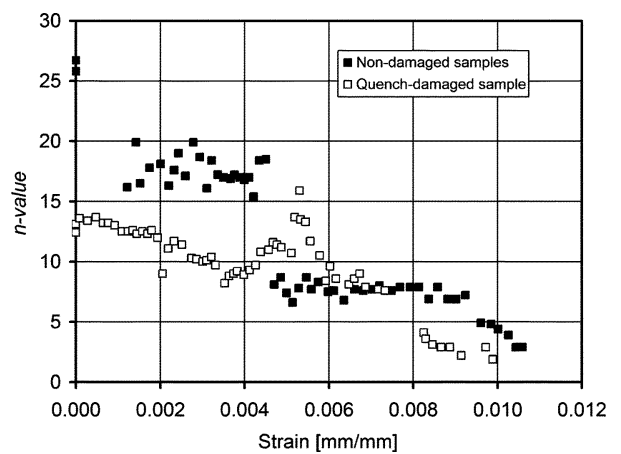

Fig. 11. $n$-values versus strain for samples that were previously subjected to $2 \times 10^{5}$ fatigue cycles.

13.6 and 25.6 for the partially quench damaged and non-damaged samples respectively. The observed $I_{c}$ and $n$-value are about the same as those before the samples were subjected to $2 \times 10^{5}$ fatigue cycles.

Fig. 12(a) shows a typical ESEM micrograph for a YBCO coated conductor sample used as a reference; this sample was obtained from the same conductor batch used in this study but was not subjected to mechanical strain or quenching. No cracks are observed. Fig. 12(b) shows a similar image from a sample that was partially quench damaged. This image is typical for the damaged samples, and also shows no cracks. Figs. 12(a) and (b) look similar and one would expect these samples to have similar electromechanical properties, but this is not the case.

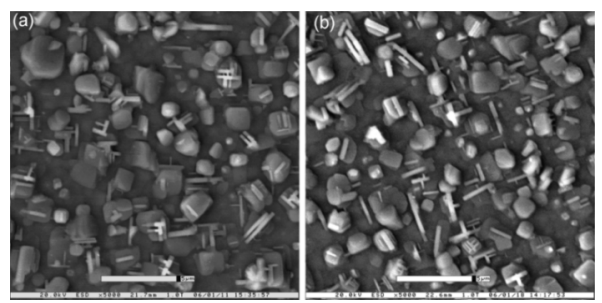

Fig. 12. ESEM micrographs of (a) a sample that was not subjected to any strain or quench, and (b) a partially quench damaged sample. No cracks are observed in either sample, indicating that quench damage does not cause cracks of sufficient size to be observed by ESEM.

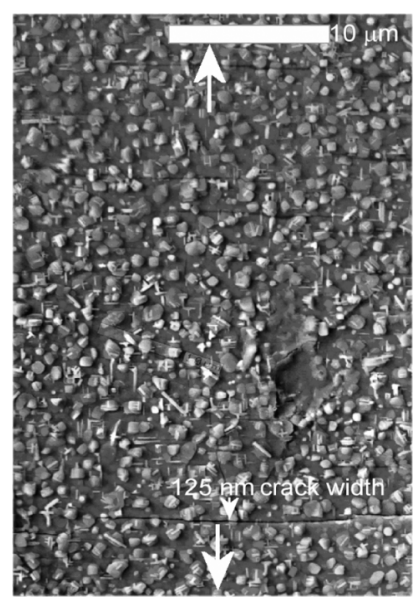

Fig. 13. Crack formation on a sample subjected to $I_{c}$-strain testing. Cracks initiate at the edges and continue to the width of the conductor. The arrows indicate the direction of the applied load.

Fig. 13 shows a typical SEM micrograph from a sample subjected to $\sim 0.103 \%$ strain. Transverse cracks cover a large area of the sample. The crack widths are relatively large $(125-250 \mathrm{~nm})$ on the edges of the sample compared to those found in the middle section $(\sim 60 \mathrm{~nm})$.

\section{DISCUSSION}

The stress-strain results for non-damaged and partially quench-damaged samples show significant differences that are attributed to the quench damage. Though the Young's moduli are same, the yield stress of the damaged sample is $85 \mathrm{MPa}$ lower. Furthermore, a closer look at the curve for the damaged sample shows a change of slope at approximately $0.15 \%$ strain which is not observed in the non-damaged sample. $I_{C}$ degraded after reaching a critical strain, which depended on the extent of the quench damage.

Hastelloy C 276, a Ni alloy, is the substrate for these YBCO conductors. Generally, nickel alloys soften when quenched from temperatures ranging from $790-1220^{\circ} \mathrm{C}$ [9]. They maybe hardened, however, by annealing above $480^{\circ} \mathrm{C}$ for a specific time, followed by furnace or air-cooling but not rapid cooling. The mechanical properties of YBCO coated conductors depend mostly on the substrate properties [10]. The peak temperature during quenching was $450 \mathrm{~K}\left(177^{\circ} \mathrm{C}\right)$, which was followed by cooling to $37 \mathrm{~K}\left(-236^{\circ} \mathrm{C}\right)$. Thus, there was both the rapid temperature increase associated with quenching the superconducting state, and a rapid cooling after the heating ended 
(typically also called a quench in materials processing nomenclature) which caused softening of the substrate. Subsequently, the superconducting sample developed spatially varying mechanical properties based on the local temperature-time history.

$I_{c}$-strain shows that the critical strain for the partially quenchdamaged sample is lower than that of non-damaged sample. The critical strain for the damaged section is $0.33 \%$ while it is $0.43 \%$ for the non-damaged section. If both samples are assumed to have the same initial $I_{c}$, then there is also a $63.8 \%$ reduction in $I_{c}$.

The fatigue testing results show no performance degradation below $95 \%$ of the initial $I_{c}$ for both samples. The partially damaged sample, however, has a slightly lower performance compared to the non-damaged sample for the entire tested range of cycles.

The $n$-values during fatigue testing remained close to initial $n$-values up to $10^{5}$ cycles where they started to decrease slowly for both samples. There was, however, no change in $I_{c}$ observed after reaching $10^{5}$ cycles. This might be the point where crack either begin to nucleate but have not grown sufficiently large to degrade $I_{c}$. It is also likely that crack formation occurs at pre-existing defects that limit current flow in their immediate vicinity even before crack nucleation. In that case, degradation will not occur until cracks grow into a region of large initial $I_{c}$ and where there is no alternative supercurrent path available.

The results for the second $I_{c}$-strain test performed on samples that were first subjected to $2 \times 10^{5}$ fatigue cycles show a trend similar to that observed in the $I_{c}$-strain for samples that were not subjected to fatigue. The quench-damaged sample crossed the $95 \% I_{c 0}$ line at $0.33 \%$ strain while the non-damaged sample did so at $0.45 \%$ strain. This is a significant difference in critical strain that must be considered, particularly in high strain applications. Since the two samples were subjected to the same fatigue conditions, it was expected that they would have similar $I_{c}$-strain behavior. The observed differences in the electromechanical properties are attributed to the quench damage.

The $n$-values obtained during $I_{c}$-strain tests are lower for the partially damaged samples than for non-damaged ones. This reflects that the $I_{c}$ for the partially damaged samples are lower that those for non-damaged samples. The $n$-values for all the samples started to decrease after the strain reached the critical values at which $I_{c}$ degradation began.

The formation of cracks from mechanical testing implies that these cracks cause $I_{c}$ degradation. Furthermore, the direction of the cracks follows the pattern (transverse to direction of applied force) of cracks that has been observed by others. The crack pattern also indicates that cracks nucleate at the sample edge and propagated inwards.

\section{Conclusions}

YBCO coated conductors with $\mathrm{Cu}$ stabilizer are degraded during quenching when the peak temperature is sufficiently high. Here, samples experienced a hot-spot temperature up to $450 \mathrm{~K}$ and $I_{c}$ was reduced locally from $220 \mathrm{~A}$ to $110 \mathrm{~A}$ at $37 \mathrm{~K}$, corresponding to a decrease in the end-to-end $I_{c}$ from 220 A to 130 A. Sections neighboring to the most degraded section also experienced $I_{c}$ degradation, but to a lesser extent. $I_{c}$ remained unchanged for sections distant from the hot spot. A spatial temperature gradient as high as $150 \mathrm{~K} / \mathrm{cm}$ and a temporal temperature gradient of $400 \mathrm{~K} / \mathrm{s}$ appear in the sample during the experiment. It remains unknown, however, whether the hot spot temperature or temperature gradient cause the local $I_{c}$ degradation.

Partial quench damage causes degradation of the mechanical properties of the superconducting tapes. Significant differences are observed in the stress-strain results. The severity of degradation depends on the extent of the quench damage. The mechanical property degradation is attributed to softening of the $\mathrm{Ni}$ alloy substrate during the rapid heating and cooling associated with the quench event.

The $n$-values for partially quench-damaged samples are less than those for non-damaged samples. This implies that the damage caused by quenching may be more complex than merely eliminating superconducting pathways within regions of the conductor. The effects on $n$-value require further study to better understand the conductor behavior.

Partial quench-damage causes degradation of the $I_{c}$-strain behavior. At $95 \% I_{c 0}$, differences of $0.1 \%-0.12 \%$ strain are observed between the damaged and the non-damaged samples.

No $I_{c}$ degradation was observed after $2 \times 10^{5}$ fatigue cycles at a strain ratio of 0.5 and a $0.35 \%$ maximum strain in either partially quench damaged and non-damaged samples. This, however, does not indicate that these samples have identical fatigue properties, since testing to more cycles may reveal some differences.

No cracks were observed in ESEM micrographs for the partially quenched damaged samples; images are similar to those from a non-damaged (virgin) sample. Thus, partial quench damages did not cause cracking in the superconductor. This confirms that the current degradation in the partially damaged samples is not due to formation of cracks but rather, degradation of superconducting properties due to the heating during quenching.

Transverse cracks have been observed on the microstructural images for samples subjected to $I_{c}$-strain tests. The formation of cracks is a result of the applied strain on the sample and is the cause of $I_{c}$ degradation observed for samples subjected to $I_{c}$-strain tests.

This study has observed that mechanical properties of YBCO coated conductors are degraded by partial quench damage. The yield stress is reduced and the $I_{c}$-strain behavior is also inferior for quench damaged sample compared to the non-damaged samples.

\section{REFERENCES}

[1] Y. Iwasa, "Stability and protection of superconducting magnets-A discussion," IEEE Trans. Appl. Supercond., vol. 15, no. 2, pp. 1615-1620, 2005.

[2] J. W. Lue, M. J. Gouge, and R. C. Duckworth, "Over-current testing of HTS tapes," IEEE Trans. Appl. Supercond., vol. 15, no. 2, pp. 1835-1838, 2005.

[3] Y. Iwasa, J. Jankowski, S.-Y. Hahn, H. Lee, J. Bascuñán, J. Reeves, A. Knoll, Y.-Y. Xie, and V. Selvamanickam, "Stability and quench protection of coated YBCO composite tape," IEEE Trans. Appl. Supercond., vol. 15, no. 2, pp. 1683-1686, 2005.

[4] F. Trillaud, H. Palanki, U. P. Trociewitz, S. H. Thompson, H. W. Weijers, and J. Schwartz, "Normal zone propagation experiments on HTS composite conductors," Cryogenics, vol. 43, pp. 271-279, 2003.

[5] X. Wang, A. R. Caruso, M. Breschi, G. Zhang, U. P. Trociewitz, H. W. Weijers, and J. Schwartz, "Normal zone initiation and propagation in $\mathrm{Y}-\mathrm{Ba}-\mathrm{Cu}-\mathrm{O}$ coated conductors with $\mathrm{Cu}$ stabilizer," IEEE Trans. Appl. Supercond., vol. 15, no. 2, pp. 2586-2589, 2005.

[6] J. Schwartz and H. W. Weijers, "Electrical measurements on superconductors by transport," in Characterization of Materials, E. N. Kaufmann, Ed. New York: John Wiley \& Sons, 2003, pp. 472-489. 
[7] A. L. Mbaruku, U. P. Trociewitz, and J. Schwartz, "Development of a low temperature electro-mechanical testing device," IEEE Trans. Appl. Supercond., vol. 15, no. 2, pp. 3620-3623, 2005.

[8] A. L. Mbaruku, "Electromechanical and Fatigue Properties of As-Manufactured and Quench Damaged YBCO Coated Conductor," $\mathrm{Ph}$. D. Dissertation, Department of Mechanical Engineering, Florida State University, Tallahassee, FL., 2006, pp.39-65.
[9] D. J. Tillack, J. M. Manning, and J. R. Hensley, "Heat treating of nickel and nickel alloys," in ASM Handbook. : : 1991, vol. 4, pp. 907-912.

[10] N. Cheggour, J. W. Ekin, C. C. Clickner, D. T. Verebelyi, C. L. H Thieme, R. Feenstra, and A. Goyal, "Reversible axial-strain effect and extended strain limits in Y-Ba-Cu-O coatings on deformation-textured substrates," Appl. Phys. Lett., vol. 83, pp. 4223-4225, 2003. 